\title{
Quantum-optical implementation of non-Hermitian potentials for asymmetric scattering
}

\author{
A. Ruschhaupt $\odot,{ }^{1}$ A. Kiely $\odot,{ }^{2}$ M. A. Simón, ${ }^{3}$ and J. G. Muga $\odot^{3}$ \\ ${ }^{1}$ Department of Physics, University College Cork, Ireland \\ ${ }^{2}$ School of Physics, University College Dublin, Belfield 4, Ireland \\ ${ }^{3}$ Departamento de Química Física, UPV/EHU, Apdo 644, 48080 Bilbao, Spain
}

(Received 4 August 2020; accepted 8 October 2020; published 5 November 2020)

\begin{abstract}
Non-Hermitian, one-dimensional potentials which are also nonlocal, allow for scattering asymmetries, namely, asymmetric transmission or reflection responses to the incidence of a particle from left or right. The symmetries of the potential imply selection rules for transmission and reflection. In particular, parity-time (PT) symmetry or the symmetry of any local potential do not allow for asymmetric transmission. We put forward a feasible quantum-optical implementation of non-Hermitian, nonlocal, non-PT potentials to implement different scattering asymmetries, including transmission asymmetries.
\end{abstract}

DOI: 10.1103/PhysRevA.102.053705

\section{INTRODUCTION}

The asymmetric response of diodes, valves, or rectifiers to input direction is of paramount importance in many different fields and technologies, from hydrodynamics to microelectronics, as well as in biological systems. We expect a wealth of applications of such response asymmetries also in the microscopic quantum realm, in particular, in circuits or operations carrying or processing quantum information with moving atoms. So far devices such as Maxwell demons, which let atoms pass one way, have been instrumental, first as ideal devices to understand the second law [1,2], and also as practical sorting devices [3-11].

Asymmetric transmission and reflection probabilities for one-dimensional (1D) particle scattering off a potential center are not possible if the Hamiltonian is Hermitian [12,13]. Non-Hermitian (NH) Hamiltonians representing effective interactions have a long history in nuclear, atomic, and molecular physics, and have become common in optics, where wave equations in waveguides could simulate Schrödinger equations [14-16]. Non-Hermitian Hamiltonians constructed by analytically continuing Hermitian ones are useful and efficient tools to find resonances [17]. They can also be set phenomenologically, e.g., to describe gain and loss [14], or be found as effective Hamiltonians for a subspace from a Hermitian Hamiltonian of a larger system by projection [12,18,19].

Much of the recent interest in Non-Hermitian Hamiltonians focuses on parity-time (PT) symmetric Hamiltonians $[20,21]$ because of their spectral properties and useful applications, mostly in optics $[15,16,22]$, but alternative symmetries are also being studied [23-31]. Symmetry operations on $\mathrm{NH}$ Hamiltonians can be systematized into group structures [26,28,29]. In particular, for 1D particle scattering

Published by the American Physical Society under the terms of the Creative Commons Attribution 4.0 International license. Further distribution of this work must maintain attribution to the author(s) and the published article's title, journal citation, and DOI. off a potential center, the different Hamiltonian symmetries imply selection rules for asymmetric transmission and reflection $[26,28] .{ }^{1}$ Whereas hermiticity does not allow for any asymmetry in transmission and reflection probabilities, PT symmetry or the symmetry of local potentials, technically "pseudohermiticy with respect to time reversal" [26], do not allow for asymmetric transmission [12,13], see symmetries II (Hermiticity), VII (PT symmetry), and VI (time-reversal pseudohermiticity) in Table I. (Here a "local potential" is defined as one whose only nonzero elements in coordinate representation are diagonal, whereas a nonlocal one has nonzero nondiagonal elements.) Thus nonlocal, non-PT, and non-Hermitian potentials are needed to implement a rich set of scattering asymmetries, and in particular, asymmetric transmission.

In this paper, we put forward a physical realization of effective NH, nonlocal Hamiltonians which do not posses PT symmetry. Nonlocal potentials for asymmetric scattering had been constructed as mathematical models [26], but a physical implementation had been so far elusive. Using Feshbach's projection technique it is found that the effective potentials for a ground-state atom crossing a laser beam in a region of space are generically nonlocal and non-Hermitian. Shaping the spatial-dependence of the, generally complex, Rabi frequency, and selecting a specific laser detuning allows us to produce different potential symmetries and asymmetric scattering effects, including asymmetric transmission.

After a lightning review of Hamiltonian symmetries and the corresponding scattering selection rules in Sec. II, we shall explain in Sec. III how to generate different NH symmetries in a quantum optical setting of an atom impinging on a laser illuminated region. Finally we provide specific example devices (constructed using numerical optimisation) with different asymmetric scattering responses in Sec. IV. Realistic experimental parameters are also examined. The asymmetric

\footnotetext{
${ }^{1}$ Throughout the paper, we assume a linear theory for systems whose wave equation is linear in the wave function.
} 
TABLE I. Conditions leading to specific symmetries in the potential (8). A given symmetry also implies others, see the last column.

\begin{tabular}{lcc}
\hline \hline Symmetry & Conditions & Implies \\
\hline (I) $1 H=H 1$ & none & - \\
(II) $1 H=H^{\dagger} 1$ & $q=-q^{*}($ i.e. $\operatorname{Re} q=0)$ & I \\
(III) $\Pi H=H \Pi$ & $\Omega(x)=e^{i \phi} \Omega(-x)$ & I \\
(IV) $\Pi H=H^{\dagger} \Pi$ & $q=-q^{*} \& \Omega(x)=e^{i \phi} \Omega(-x)$ & III, II, I \\
(V) $\Theta H=H \Theta$ & $q=-q^{*} \& \Omega(x)=e^{i \phi} \Omega(x)^{*}$ & VI, II, I \\
(VI) $\Theta H=H^{\dagger} \Theta$ & $\Omega(x)=e^{i \phi} \Omega(x)^{*}$ & I \\
(VII) $\Theta \Pi H=H \Theta \Pi$ & $q=-q^{*} \& \Omega(x)=e^{i \phi} \Omega(-x)^{*}$ & VIII, II, I \\
(VIII) $\Theta \Pi H=H^{\dagger} \Theta \Pi$ & $\Omega(x)=e^{i \phi} \Omega(-x)^{*}$ & I \\
\hline \hline
\end{tabular}

behavior can be intuitively understood based on a classical approximation of the motion and the noncommutativity of rotations on the Bloch sphere, which gives good estimates for the potential parameters, see Sec. V.

\section{SYMMETRIES OF SCATTERING HAMILTONIANS}

We consider one-dimensional scattering Hamiltonians $H=H_{0}+V$, where $H_{0}$ is the kinetic energy for a particle of mass $m$, and $V$ is the potential, which is assumed to decay fast enough on both sides so that $H$ has a continuous spectrum and scattering eigenfunctions. These eigenfunctions may be chosen so that asymptotically, i.e., far from the potential center, they are superpositions of an incident plane wave and a reflected plane wave on one side, and a transmitted plane wave on the other side. Reflected and transmitted waves include corresponding amplitudes, whose squared-modulii (scattering coefficients hereafter) sum to one for Hermitian potentials. Instead, $\mathrm{NH}$ potentials may produce absorption or gain.

There are eight different symmetries that $H$ could fulfill, see Table I, with the forms

$$
\begin{aligned}
& A H=H A, \\
& A H=H^{\dagger} A,
\end{aligned}
$$

where $A$ is a unitary or antiunitary operator in the Klein fourgroup $K_{4}=\{1, \Pi, \theta, \Pi \theta\}$ [26]. Relation (2) is called here $A$ pseudohermiticity of $H[26,32]$. The operators $1, \Pi, \theta$ and $\Pi \theta$ are the identity, parity, time reversal, and the consecutive (commuting) application of both operators. Acting on position eigenvectors $|x\rangle, \Pi c|x\rangle=c|-x\rangle$, and $\theta c|x\rangle=c^{*}|x\rangle$, for any complex number $c$. Note that symmetry I is a trivial symmetry and is satisfied for all Hamiltonians.

The eight symmetries may be regarded as the invariance of the Hamiltonian with respect to eight symmetry operations that form the Abelian group E8 [28]. They are all operations that can be done by inversion, transposition, complex conjugation, and their combinations. Making use of generalized unitarity relations and the relations implied by the symmetries on $S$-matrix elements, the transmission and reflection amplitudes for right and left incidence, $T^{r}, R^{r}$ and $T^{l}, R^{l}$, can be related to each other, as well as their modulii [26]. "Right and left incidence" are here shorthands for "incidence from the right" and "incidence from the left," respectively.

The possible asymmetric responses are allowed or forbidden, according to selection rules, by the symmetries of the
TABLE II. Device types for transmission and/or reflection asymmetry in the first row (see main text for nomenclature, binary values ( 0 or 1 ) for the transmission and reflection coefficients are considered here as an ideal case). The second row gives the corresponding symmetries that allow each device.

\begin{tabular}{lccccc}
\hline \hline $\mathcal{T R} / \mathcal{A}$ & $\mathcal{T} / \mathcal{R}$ & $\mathcal{T} / \mathcal{A}$ & $\mathcal{T} \mathcal{R} / \mathcal{R}$ & $\mathcal{R} / \mathcal{A}$ & $\mathcal{T} \mathcal{R} / \mathcal{T}$ \\
\hline $\mathrm{I}$ & $\mathrm{I}$ & I,VIII & I,VIII & I,VI & I, IV, VI, VII \\
\hline \hline
\end{tabular}

Hamiltonian. If we impose that the transmission and reflection coefficients have only 0 or 1 values, a convenient reference scenario for devices intended to manage quantum-information applications, six possible scattering asymmetries may be identified [26], see Table II. It is useful to label them according to the response to incidence from the left or right. The possible responses are encoded in the letters $\mathcal{A}$, for "absorption', and $\mathcal{T}$ and $\mathcal{R}$ for "transmission" and "reflection" separated by "/". The letters on the left of / are for left incidence, and the ones on the right are for right incidence. For example, $\mathcal{T} / \mathcal{A}$ means transmission for left incidence and absorption for right incidence. From the selection rules [26], it is possible to determine which symmetries allow for a given device, see Table II.

The relations between the symmetries and "reciprocity" are surely worth spelling out, in view of many works and discussions in optics [33-36]. "Reciprocity" is a somewhat vague term with different meanings for different authors and communities, the reviews [37] and [38] give some useful background. A primary formulation regards reciprocity as the property of detecting the same effects when interchanging source and detector without changing the scatterer. This concept has lead to different formalizations that fix in more detail what is exactly meant by "same effects" and "interchanging source and detector." In 1D scattering problems, we may first distinguish a reciprocity for scattering amplitudes or for scattering coefficients (their modulus squared). We shall hereafter focus on coefficients as in the rest of the paper. Another distinction can be made between reflection and transmission reciprocities, namely, a system with $\left|R^{l}\right|^{2}=\left|R^{r}\right|^{2}$ would be "reflection reciprocal" and if $\left|T^{l}\right|^{2}=\left|T^{r}\right|^{2}$ the system would be transmission reciprocal. ${ }^{2}$ A formal definition of reciprocity is that, for some antiunitary operator $K$ [38],

$$
H K=K H^{\dagger} \text {. }
$$

It follows that the scattering transition matrix obeys in momentum representation [38]

$$
\left\langle p|\mathrm{~T}| p^{\prime}\right\rangle=\left\langle K p^{\prime}|\mathrm{T}| K p\right\rangle .
$$

In our symmetry classification, symmetries VI and VIII obey by definition reciprocity conditions of the form (3). Inserting the results in the exact forms of transmission and reflection amplitudes, which depend on diagonal and nondiagonal elements of the transition matrix, respectively, see, e.g., Ref. [12]

\footnotetext{
${ }^{2}$ Incidentally, for reflection reciprocity, "interchanging source and detector" has to be understood in momentum space rather than spatially.
} 
or the Supplementary Material, different physical consequences follow. in symmetry VI, $K=\Theta,|K p\rangle=|-p\rangle$, and the reciprocity condition implies transmission reciprocity. In symmetry VIII, $K=\Pi \Theta$ and $|K p\rangle=|p\rangle$, so the reciprocity condition implies reflection reciprocity. A first relevant observation is this: an arbitrary reciprocity condition of the form (3), does not necessarily imply symmetrical transmission. A second point is that "scattering selection rules," i.e., the set of forbidden phenomena, or compulsory relations among right and left coefficients, see Table I in Ref. [26], depend as well on generalized unitary relations. Putting together the effect of symmetries on transition or $S$-matrix elements and generalized unitarity relations, it turns out that symmetries II (Hermiticity) and III (parity) are not capable of any, reflection or transmission, asymmetry; symmetries VI (time reversal pseudohermiticity) and VII (PT symmetry) allow for reflection asymmetry but not for transmission asymmetry; symmetries V (time-reversal symmetry) and VIII (PT pseudohermiticity) allow for transmission asymmetry but not for reflection asymmetry, whereas I (trivial symmetry) and IV (parity pseudohermiticity) allow for both scattering asymmetries. Note also the importance on nonlocality for asymmetric transmission: All local potentials do satisfy automatically symmetry VI, and are therefore necessarily transmission reciprocal. Let us insist once more than all these results are for linear (Schrödinger) dynamics. Nonlinearity allows to break down these selection rules $[33,36,39]$.

\section{EFFECTIVE NONLOCAL POTENTIAL FOR THE GROUND STATE OF A TWO-LEVEL ATOM}

The key task is now to physically realize some of the potential and device types described in the previous section. We start with a two-level atom with ground level $|1\rangle$ and excited state $|2\rangle$ impinging onto a laser illuminated region. For a full account of the model and further references see [40]. The motion is assumed one dimensional, either because the atom is confined in a waveguide or because the direction $x$ is uncoupled to the others. We only account explicitly for atoms before the first spontaneous emission in the wavefunction [41-43]. If the excited atom emits a spontaneous photon it disappears from the coherent wavefunction ensemble. We assume that no resetting into the ground state occurs. The physical mechanism may be an irreversible decay into a third level [44], or atom ejection from the waveguide or the privileged 1D direction due to random recoil [45]. The state $\boldsymbol{\Phi}_{k}=\left(\begin{array}{c}\phi_{k}^{(1)} \\ \phi_{k}^{(2)}\end{array}\right)$ for the atom before the first spontaneous emission impinging with wave number $k$ in a laser adapted interaction picture, obeys, after applying the rotating wave approximation, an effective stationary Schrödinger equation with a time-independent Hamiltonian $[19,40] \mathcal{H} \boldsymbol{\Phi}_{k}(x)=E \boldsymbol{\Phi}_{k}(x)$, where

$$
\begin{aligned}
\mathcal{H} & =H_{0} \mathbf{1}+\mathcal{V}=\frac{1}{2 m}\left(\begin{array}{cc}
p^{2} & 0 \\
0 & p^{2}
\end{array}\right)+\mathcal{V}(x), \\
\mathcal{V}(x) & =\frac{\hbar}{2}\left(\begin{array}{cc}
0 & \Omega(x) \\
\Omega(x)^{*} & -(2 \Delta+i \gamma)
\end{array}\right) .
\end{aligned}
$$

We assume perpendicular incidence of the atom on the laser sheet for simplicity, oblique incidence is treated, e.g., in Refs. $[6,40]$. Here $E=\hbar^{2} k^{2} / 2 m$ is the energy, and $\Omega(x)$ is the position-dependent, on-resonance Rabi frequency, where real and imaginary parts may be controlled independently using two laser field quadratures [46]; $\gamma$ is the inverse of the life time of the excited state; $\Delta=\omega_{L}-\omega_{12}$ is the detuning (laser angular frequency minus the atomic transition angular frequency $\left.\omega_{12}\right) ; p=-i \hbar \partial / \partial x$ is the momentum operator; and $\mathbf{1}=|1\rangle\langle 1|+| 2\rangle\langle 2|$ is the unit operator for the internal-state space. Complementary projectors $P=|1\rangle\langle 1|$ and $Q=|2\rangle\langle 2|$ are defined to select ground and excited state components. Using the partitioning technique $[18,47,48]$, we find for the ground state amplitude $\phi_{k}^{(1)}$ the equation

$$
E \phi_{k}^{(1)}(x)=H_{0} \phi_{k}^{(1)}(x)+\int d y\langle x, 1|\mathcal{W}(E)| y, 1\rangle \phi_{k}^{(1)}(y),
$$

where $\mathcal{W}(E)=P \mathcal{V} P+P \mathcal{V} Q(E+i 0-Q \mathcal{H} Q)^{-1} Q \mathcal{V} P, \quad$ is generically non local and energy dependent. Specifically, we have now achieved a physical realization of an effective (in general) nonlocal, non-Hermitian potential whose kernel has the form

$$
V(x, y)=\langle x, 1|\mathcal{W}(E)| y, 1\rangle=\frac{m}{4} \frac{e^{i|x-y| q}}{i q} \Omega(x) \Omega(y)^{*},
$$

where $q=\frac{\sqrt{2 m E}}{\hbar}(1+\mu)^{1 / 2}, \operatorname{Im} q \geqslant 0, \quad$ and $\quad \mu=\frac{2 \Delta+i \gamma}{2 E / \hbar}$. Eq. (8) is worked out in momentum representation to do the integral using the residue theorem. This is a generalized, nonlocal version of the effective potentials known for the ground state $[44,49]$, which are found from Eq. (8) in the large $\mu$ limit [19]. The reflection and transmission amplitudes $R^{r, l}$ and $T^{r, l}$ may be calculated directly using the potential (8) or as corresponding amplitudes for transitions from ground state to ground state in the full two-level theory (see Appendix).

\section{A. Possible symmetries of the nonlocal potential}

The necessary conditions for the different symmetries of the potential (8) are outlined in the second column of Table I. For example, symmetry III (parity) requires that $V(x, y)=$ $V(-x,-y)$ [26]. Inserting the functional form of the potential from Eq. (8) into this condition, it results in the requirement $\Omega(x) \Omega(y)^{*}=\Omega(-x) \Omega(-y)^{*}$. This is fulfilled if $\Omega(x)=$ $\Omega(-x) e^{i \phi}$ with some arbitrary phase freedom $\phi$.

Since $\Omega(x)$ does not depend on $q$, symmetries IV, $\mathrm{V}$ and VII imply that symmetry II is obeyed as well (Hermiticity). Moreover symmetry III (parity) should be discarded for our purpose since it does not allow for asymmetric transmission or reflection [26]. This leaves us with three interesting symmetries to explore: VI, which allows for asymmetric reflection; VIII which allows for asymmetric transmission, and I, which in principle allows for arbitrary asymmetric responses, except for physical limitations imposed by the two-level model (see Appendix).

As seen from Table I, $\operatorname{Re}(q)=0$ makes the potential Hermitian so we shall avoid this condition. If $\gamma=0, \mu \in$ $\mathbb{R}$. Hence $\mu+1<0$ gives $\operatorname{Re}(q)=0$ and $\mu+1>0$ gives $\operatorname{Im}(q)=0 \cdot \mu+1>0$ amounts to a condition on the detuning compared to the incident energy, namely $\Delta>-E / \hbar$. In the following examples, we implement potentials with symmetries VIII, VI, and I, with detunings and energies satisfying the condition $\mu+1>0$. 

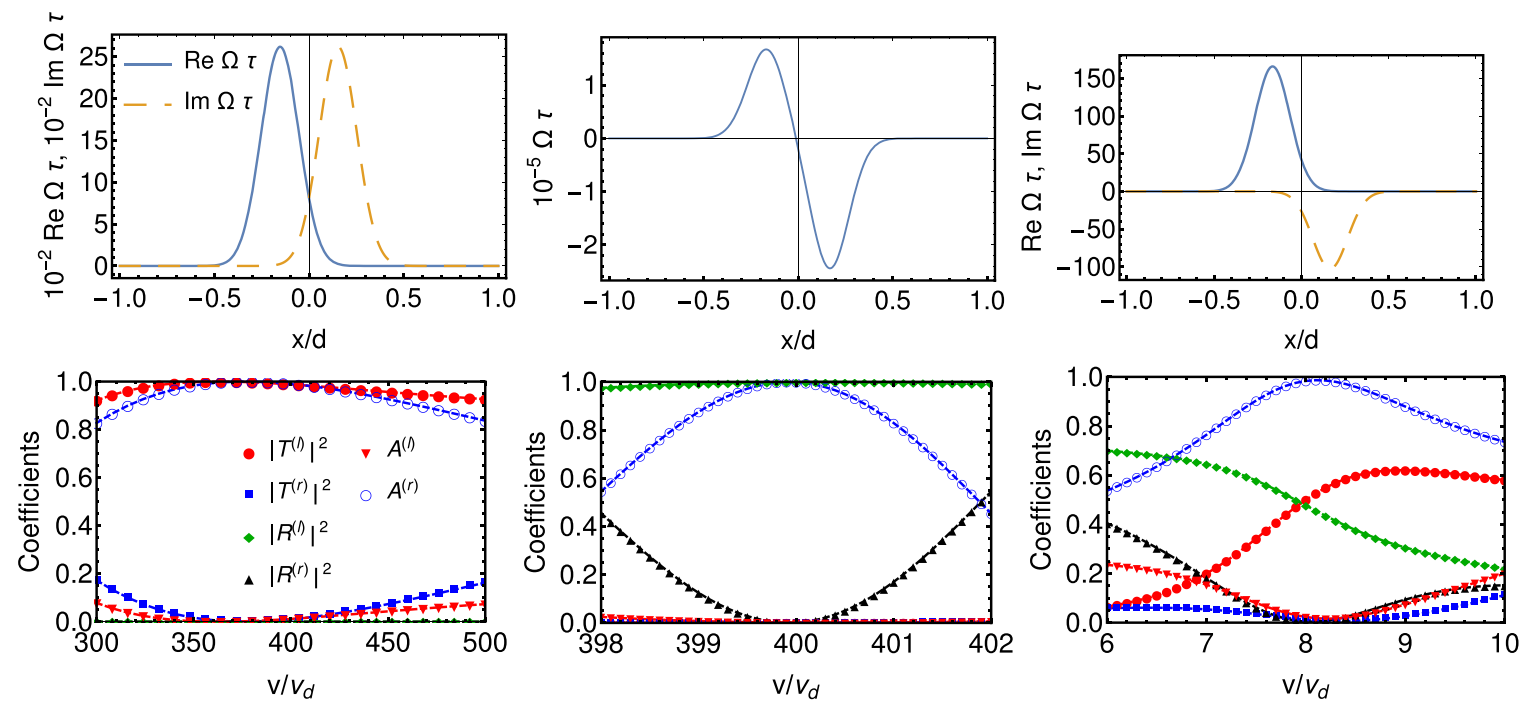

FIG. 1. Left column: $\mathcal{T} / \mathcal{A}$ device with symmetry VIII. (Top) $\Omega_{\mathrm{VIII}}(x)$. (Bottom) Transmission and reflection coefficients. $v_{0} / v_{d}=400$, $a \tau=2618.19, x_{0} / d=0.1532$, and $\tau \Delta=1413.01$. (Middle column) $\mathcal{R} / \mathcal{A}$ device with symmetry VI. (Top) $\Omega_{\mathrm{VI}}(x)$ (it is real). (Bottom) Transmission and reflection coefficients. $v_{0} / v_{d}=400, b \tau=-244516.1, c \tau=167853.9, x_{0} / d=0.1679$, and $\tau \Delta=193.508$. (Right column) "Partial" $-\mathcal{T} \mathcal{R} / \mathcal{A}$ device with symmetry I. (Top) $\Omega_{\mathrm{I}}(x)$, real (blue, solid line) and imaginary parts (orange, dashed line). (Bottom) Transmission and reflection coefficients. $v_{0} / v_{d}=8, b \tau=102.6520, c \tau=165.8355, x_{0} / d=0.1648$, and $\tau \Delta=90.5337$. In all cases, $\tau=m d^{2} / \hbar$ and $v_{d}=$ $\hbar /(m d)$.

\section{DESIGN OF ASYMMETRIC DEVICES}

We will now apply this method to physically realize nonlocal potentials of the form (8). We shall work out explicitly a $\mathcal{T} / \mathcal{A}$ device with symmetry VIII, a $\mathcal{R} / \mathcal{A}$ device with symmetry VI, and a "partial" $-\mathcal{T} \mathcal{R} / \mathcal{A}$ device, having $1 / 2$ transmission and reflection coefficients from the left, with symmetry I. The $\mathcal{T} / \mathcal{A}$ and the "partial" $-\mathcal{T} \mathcal{R} / \mathcal{A}$ device have transmission asymmetry so they cannot be built with local or $P T$-symmetric potentials. Let us motivate the effort with some possible applications, relations and analogies of these devices. $\mathcal{T} / \mathcal{A}$ and $\mathcal{R} / \mathcal{A}$ are, respectively, transmission and reflection filters. They are analogous to half-wave electrical rectifiers that either let the signal from one side "pass" (transmitted) or change its sign (reflected) while suppressing the other half signal. They may play the role of half-rectifiers in atomtronic circuits. A $\mathcal{T} / \mathcal{A}$ device allows us, for example, to empty a region of selected particles, letting them go away while not letting particles in. The "atom diode" devices worked out, e.g., in Refs. [3-6] where of type $\mathcal{R} / \mathcal{A}$. As the mechanism behind them was adiabatic, a broad range of momenta with the desired asymmetry could be achieved. In comparison the current approach is not necessarily adiabatic so it can be adapted to faster processes.

As for the "partial"- $\mathcal{R} \mathcal{T} / \mathcal{A}$ device, it reflects and transmits from one side while absorbing from the other side. In an optical analogy, an observer from the left perceives it as a darkish mirror. An observer from the right "sees" the other side because of the allowed transmission but cannot be seen from the left since nothing is transmitted from right to left. Our device is necessarily "partial" one as there cannot be net probability gain because of the underlying two-level system, and a "full" version with both reflection and transmission coefficients equal to one would need net gain.
The three devices are worked out for $\gamma=0$, a valid approximation for hyperfine transitions. We assume for the Rabi frequencies the forms

$$
\begin{aligned}
\Omega_{\mathrm{VIII}}(x) & =a\left[g\left(x+x_{0}\right)+i g\left(x-x_{0}\right)\right], \\
\Omega_{\mathrm{VI}}(x) & =b g\left(x+x_{0}\right)+c g\left(x-x_{0}\right), \\
\Omega_{\mathrm{I}}(x) & =-i b g\left(x+x_{0}\right)+c g\left(x-x_{0}\right),
\end{aligned}
$$

in terms of smooth, realizable Gaussians $g(x)=$ $\exp \left[-x^{2} / w^{2}\right]$. We fix $2 d$ as an effective finite width of the potential area beyond which the potential is negligible and assumed to vanish. We will express in the following the different length parameters as a multiple of $d$ to keep results general. In addition, we will use as a scaling factor for the velocity $v_{d}=\hbar /(m d)$, and for time $\tau=m d^{2} / \hbar$.

In the following calculations, we fix the width of the Gaussians to be $w=\sqrt{2} d / 10$. We always first set a target velocity $v_{0}$ to achieve the desired asymmetric scattering response. The real parameters $a, b, c, x_{0}$ in Eq. (9), and $\Delta$ are then numerically optimized with the GRAPE (Gradient Ascent Pulse Engineering) algorithm [50,51].

The Rabi frequencies will fulfill the indicated symmetries VIII, VI, and I. $\Omega_{\mathrm{VI}}(x)$ should not be even (i.e., $b \neq c$ ) to avoid symmetry II. In addition, $\Omega_{\mathrm{I}}(x)$ should not fulfill any other symmetry than I. The corresponding Rabi frequencies $\Omega(x)$ are depicted in Fig. 1, top row. The scattering coefficients are shown in the bottom row. Figure 1 demonstrates that the three potentials satisfy the asymmetric response conditions imposed at the selected velocity and also in a region nearby.

The "partial" $-\mathcal{T} \mathcal{R} / \mathcal{A}$ device fullfills $\left|T^{l}\right|^{2}=\left|R^{l}\right|^{2}=1 / 2$ and full absorption from the right. The potential we use for that device has symmetry I only, i.e., "no symmetry" other than the trivial commutation with the identity. No other potential symmetry would allow this type of device. 

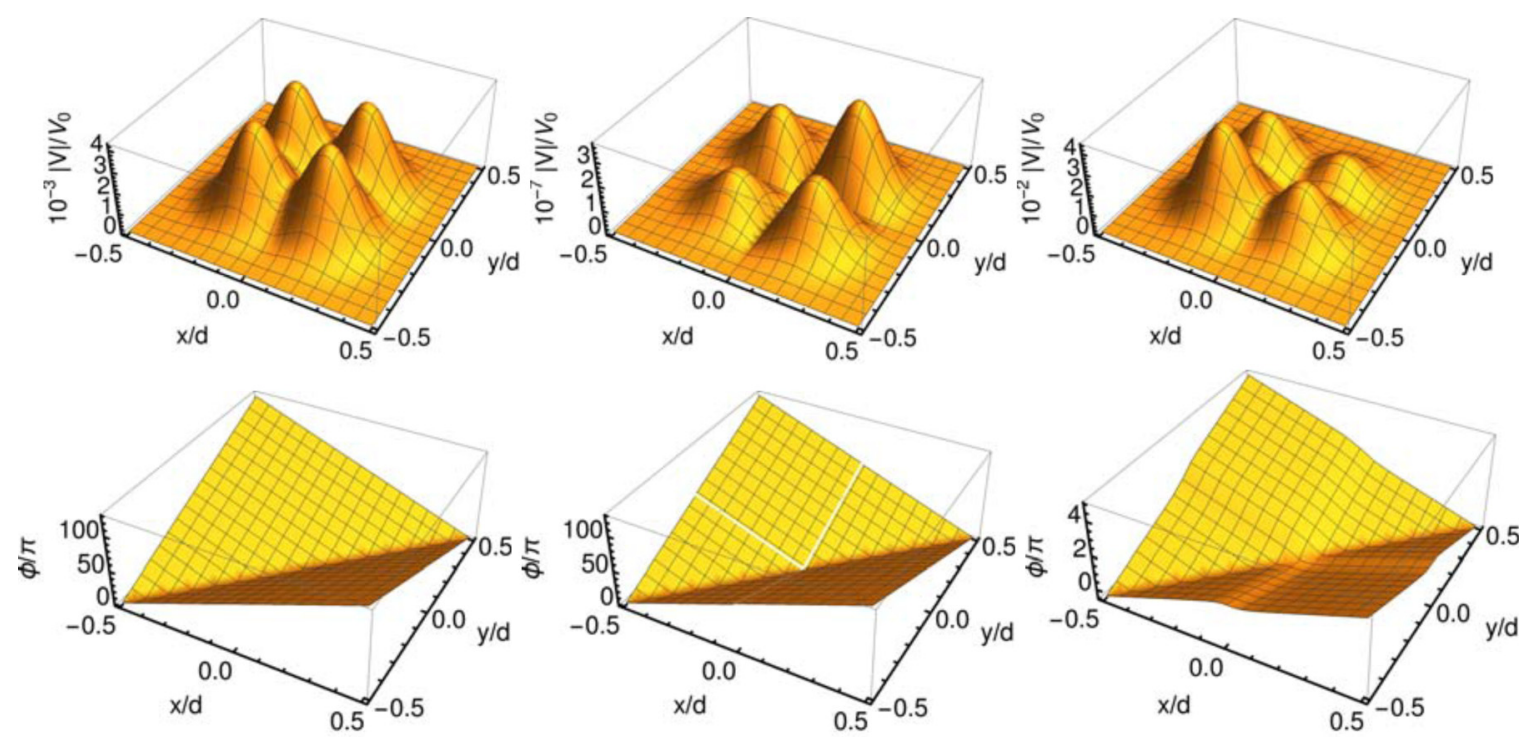

FIG. 2. Nonlocal potentials $V(x, y)$ : absolute value (top), argument (bottom). (Left column) Potential for $\mathcal{T} / \mathcal{A}$ device with symmetry VIII. (Middle column) Potential for $\mathcal{R} / \mathcal{A}$ device with symmetry VI. (Right column) "Partial" $-\mathcal{T} \mathcal{R} / \mathcal{A}$ device with symmetry I. $V_{0}=\hbar^{2} /\left(m d^{3}\right)$.

The effective nonlocal potential $V(x, y)$, see Eq. (8), corresponding to the $v / v_{d}$ ratios used for the three devices is shown in Fig. 2. Note that the nonlocal potential has dimensions energy/length, so we divide the absolute value by a factor $V_{0}=\hbar^{2} /\left(m d^{3}\right)$ to plot a dimensionless quantity.

In the parameter optimization, we see that increasing the velocities further does not pose a problem for the $\mathcal{T} / \mathcal{A}$ device, it is more challenging for a $\mathcal{R} / \mathcal{A}$ device, and it is quite difficult for the partial- $\mathcal{R} \mathcal{T} / \mathcal{A}$ device. The device $\mathcal{T} / \mathcal{A}$ is feasible for an experimental implementation as the ratio $v_{0} / v_{d}$ can be easily increased to desired values, for reasonable values of the Rabi frequency and laser waist [52].

Moreover the velocity width with the desired behavior is much broader for $\mathcal{T} / \mathcal{A}$. Therefore a $\mathcal{T} / \mathcal{A}$ device is the best candidate for an experimental implementation. As a check of feasibility, let us assume a Beryllium ion. Its hyperfine structure provides a good two-level system for which we can neglect decay (i.e., $\gamma \approx 0$ is indeed realistic). We have $m=$ $1.49 \times 10^{-26} \mathrm{~kg}$ and set a length $d=10 \mu \mathrm{m}$ compatible with the small laser waists (in this case $1.4 \mu \mathrm{m}$ ) achieved for individual ion addressing [52]. The scaling factors take the values

$$
\begin{aligned}
v_{d} & =0.67 \mathrm{~mm} / \mathrm{s}, \\
\tau & =1.49 \times 10^{-2} \mathrm{~s},
\end{aligned}
$$

which gives $v \approx 27 \mathrm{~cm} / \mathrm{s}$ for $v / v_{d}=400$ (again, we see no major obstacle to get devices for higher velocities, in particular, the classical approximations in Sec. V can be used to estimate the values of the parameters) and Rabi frequencies, see Fig. 1, in the hundreds of $\mathrm{kHz}$ range. The relative ion-laser beam velocity could be as well implemented by moving the beam in the laboratory frame.

\section{CLASSICAL APPROXIMATION FOR $\mathcal{T} / \mathcal{A}$ DEVICE}

In a $\mathcal{T} / \mathcal{A}$ device such as the one presented an incident plane wave from the left ends up as a pure transmitted wave with no reflection or absorption. However, a wave incident from the right is fully absorbed. How can that be? Should not the velocity-reversed motion of the transmitted wave lead to the reversed incident wave? For a more intuitive understanding we may seek help in the underlying two-level model. In the larger space, the potential is again local and Hermitian. A simple semiclassical approximation is to assume that the particle moves with constant speeds $\pm v$ for left $(v>0)$ or right $(-v<0)$ incidence, so that at a given time it is subjected to the $2 \times 2$ time-dependent potentials $\mathcal{V}( \pm v t)$. The incidence from the left and right give different time dependencies for the potential. The scattering problem then reduces to solving the time-dependent Schrödinger equation for the amplitudes of a two-level atom with time-dependent potential, i.e., to solving the following time-dependent Schrödinger equation $(\gamma=0)$

$$
i \hbar \frac{\partial}{\partial t} \chi_{ \pm}(t)=\mathcal{V}( \pm v t) \chi_{ \pm}(t)
$$

with the appropriate boundary conditions $\chi_{+}(-\infty)=$ $\chi_{-}(-\infty)=\left(\begin{array}{l}1 \\ 0\end{array}\right)$. The solutions for $v / v_{d}=400$ are shown in Fig. 3. In Fig. 3(a), $\chi_{+}(t)$ (left incidence) is depicted: the particle ends with high probability in the ground state at final time. In Fig. 3(b), $\chi_{-}(t)$ (right incidence) demonstrates the ground-state population is transferred to the excited state.
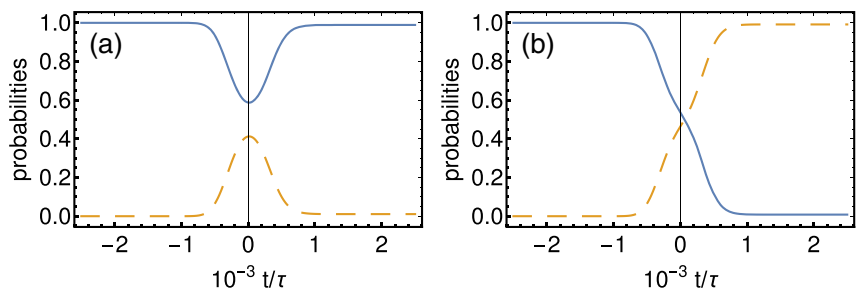

FIG. 3. Simplified model of the asymmetric $\mathcal{T} / \mathcal{A}$ device with symmetry VIII: (a) $\chi_{+}(t)$, (b) $\chi_{-}(t)$; ground-state population $\left|\chi_{ \pm(t), 1}\right|^{2}$ (blue, solid line), excited- $\left|\chi_{ \pm(t), 2}\right|^{2}$ (orange, dashed line). $v / v_{d}=400, a \tau=2618.19, x_{0} / d=0.1532$, and $\tau \Delta=1413.01$. 
Projected onto the ground-state level alone, this corresponds to full absorption of the ground-state population at final time.

For an even rougher but also illustrative picture, again in a semiclassical time-dependent framework, we may substitute the smooth Gaussians for $\operatorname{Re}(\Omega)$ and $\operatorname{Im}(\Omega)$ in Fig. 1 by two simple, contiguous square functions of height $\Omega>0$ and width $\tilde{w}>0$. Then, the $2 \times 2$ potential at a given time is, in terms of Pauli matrices,

$$
\mathcal{V}(x)=\frac{\hbar}{2} \Delta\left(\sigma_{Z}-\mathbf{1}\right)+\frac{\hbar}{2}\left\{\begin{array}{cc}
\Omega \sigma_{X} & -\tilde{w}<x<0 \\
-\Omega \sigma_{Y} & 0<x<\tilde{w} \\
0 & \text { otherwise }
\end{array}\right.
$$

where $x= \pm v t$ and let $\mathrm{T}=2 \tilde{w} / v$.

The time evolution of this process, $\chi_{ \pm}(t)$, up to a phase factor may be regarded as two consecutive rotations $R_{j}=$ $e^{-i \beta \mathbf{n}_{j} \cdot \boldsymbol{\sigma} / 2}(j=1,2)$, with $\beta=\frac{\mathrm{T}}{2} \sqrt{\Omega^{2}+\Delta^{2}}$, of the two-level state on the Bloch sphere about the axes

$$
\begin{aligned}
& \mathbf{n}_{1}=\frac{1}{\sqrt{\Omega^{2}+\Delta^{2}}}(\Omega, 0, \Delta), \\
& \mathbf{n}_{2}=\frac{1}{\sqrt{\Omega^{2}+\Delta^{2}}}(0,-\Omega, \Delta) .
\end{aligned}
$$

The initial state at time $t=-\mathrm{T} / 2$ is again $\chi_{+}(-\mathrm{T} / 2)=$ $\chi_{-}(-\mathrm{T} / 2)=\left(\begin{array}{l}1 \\ 0\end{array}\right)$. The unitary time-evolution operator to reach the final time $\mathrm{T} / 2$ takes the form $e^{i \Delta \mathrm{T} / 2} R_{2} R_{1}$ for incidence from the left $\left(\chi_{+}\right)$and $e^{i \Delta \mathrm{T} / 2} R_{1} R_{2}$ for incidence from the right $\left(\chi_{-}\right)$. The time $\mathrm{T}$ and the parameters $\Omega, \Delta$ will be fixed to reproduce the results of the full calculation with the exact model, namely, so that the system starts in the ground state to end either in the ground state $\left(\left|\chi_{+}(T / 2)\right|^{2}=1\right)$ or in the excited state by performing the rotations in one order or the reverse order $\left(\left|\chi_{-}(\mathrm{T} / 2)\right|^{2}=0\right)$. This gives $\Omega / \Delta=\sqrt{2}$ and $\mathrm{T}=4 \pi /(3 \sqrt{3} \Delta)$. It follows that $\mathbf{n}_{1}=\frac{1}{\sqrt{3}}(\sqrt{2}, 0,1)$ and $\mathbf{n}_{2}=\frac{1}{\sqrt{3}}(0,-\sqrt{2}, 1)$.

The different outcomes can thus be understood as the result of the noncommutativity of rotations on the Bloch sphere, see Fig. 4. In Fig. 4(a), first the rotation $R_{1}(\mathrm{~T} / 2)$ and then the rotation $R_{2}(\mathrm{~T} / 2)$ are performed. Starting in the ground state $|1\rangle$, the system ends up in the excited state $|2\rangle$. In Fig. 4(b), first the rotation $R_{2}(\mathrm{~T} / 2)$ and then the rotation $R_{1}(\mathrm{~T} / 2)$ are performed: now the system starts and ends in the ground state $|1\rangle$.

These results can be even used to approximate the parameters of the potential in the quantum setting. As an approximation of the height $a$ we assume that the area $a \int_{-\infty}^{\infty} d x g(x)=$ $a \sqrt{\pi} w$ is equal to $\tilde{w} \Omega=\mathrm{T} v_{0} \Omega / 2=v_{0} \pi(2 / 3)^{3 / 2}$. This results in an approximation $a \approx \frac{v_{0}}{w} \sqrt{\pi}(2 / 3)^{3 / 2}$. As an additional approximation, we assume that $(a / \sqrt{2}) / \Delta \approx \Omega / \Delta=\sqrt{2}$, so we get $\Delta \approx a / 2 \approx \frac{v_{0}}{2 w} \sqrt{\pi}(2 / 3)^{3 / 2}$. A comparison between these approximations and the numerically achieved parameters, see Fig. 5, shows a good agreement over a large velocity range. This allows one to find good initial values for further numerical optimization.

\section{DISCUSSION}

Non-Hermitian Hamiltonians display many interesting phenomena which are impossible for a Hermitian Hamilto- (a) Order of rotations: first $R_{1}(\mathrm{~T} / 2)$ (left figure) and then $R_{2}(\mathrm{~T} / 2)$ (right figure)
|1)

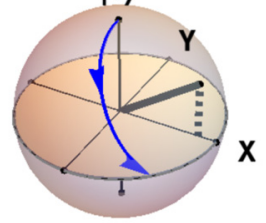

|2)

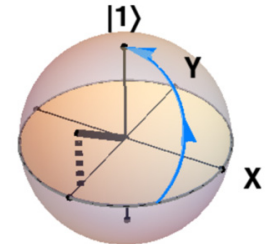

|2) (b) Order of rotations: first $R_{2}(\mathrm{~T} / 2)$ (left figure) and then $R_{1}(\mathrm{~T} / 2)$ (right figure).

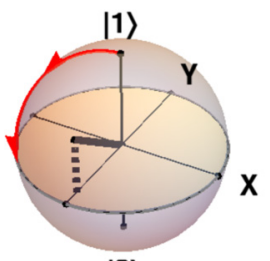

|2)

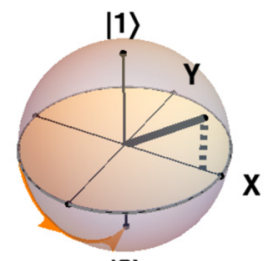

|2〉
FIG. 4. Simplified time-dependent model of the asymmetric $\mathcal{T} / \mathcal{A}$ device with symmetry VIII: Bloch sphere explaining nontime-reversal invariance, see text for details. The state trajectories are depicted in two-steps on the sphere. The rotation axes are also depicted. (a) The process simulates incidence from the left. The state starts and ends in $|1\rangle$. (b) The process simulates incidence from the right. The state starts at $|1\rangle$ and ends at $|2\rangle$.

nian acting on the same Hilbert space. In particular, in the Hilbert space of a single, structureless particle on a line formed by square integrable normalizable functions, Hermitian Hamiltonians do not allow, within a linear theory, for asymmetric scattering transmission and reflection coefficients. However, non-Hermitian Hamiltonians do. Since devices of technological interest, such as one-way filters for transmission or reflection, one-way barriers, one-way mirrors, and others, may be built based on such scattering response asymmetries, there is both fundamental interest and applications in sight to implement Non-Hermitian scattering Hamiltonians. This paper is a step forward in that direction, specifically we propose a quantum-optical implementation of potentials with asymmetric scattering response. They are nonlocal and non-PT symmetrical, which allows for asymmetric transmission.
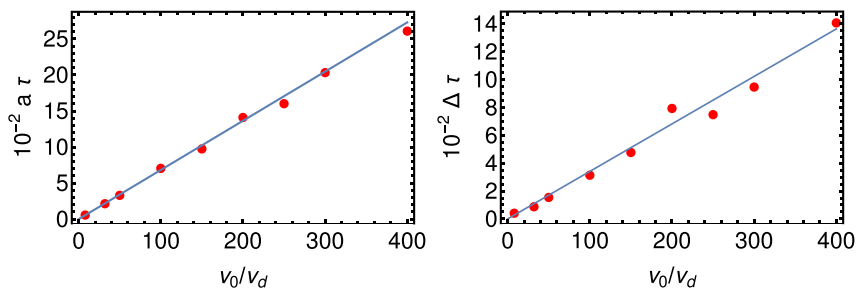

FIG. 5. Asymmetric $\mathcal{T} / \mathcal{A}$ device with symmetry VIII: comparison between numerically achieved parameters (red dots) and approximated parameters (blue, solid lines) vs velocity $v_{0}$. (a) Height of Rabi frequency $a$, (b) detuning $\Delta$. 
In general, the chosen Hilbert space may be regarded as a subspace of a larger space. For example, the space of a "structureless particle" in 1D is the ground-state subspace for a particle with internal structure, consisting of two levels in the simplest scenario. It is then possible to regard the NonHermitian physics in the reduced space as a projection of the larger space, which may itself be driven by a Hermitian or a Non-Hermitian Hamiltonian. We have seen the Hermitian option in our examples, where we assumed a zero decay constant, $\gamma=0$, for the excited state. a nonzero $\gamma$ implies a Non-Hermitian Hamiltonian in the larger two-level space. The description may still be enlarged, including quantized field modes to account for the atom-field interaction with a Hermitian Hamiltonian. As an outlook, depending on the application, there might be the need for a more fundamental and detailed descriptive level. Presently we discuss the desired physics (i.e., the scattering asymmetries) at the level of the smallest 1D space of the ground state, while taking refuge in the two-level space to find a feasible physical implementation.

\section{ACKNOWLEDGMENTS}

We acknowledge R. F. Snider and C. G. Hegerfeldt for many discussions. This work was supported by the Basque Country Government (Grant No. IT986-16), and by PGC2018-101355-B-I00 (MCIU/AEI/FEDER,UE). A.K. acknowledges financial support from the Science Foundation Ireland Starting Investigator Research Grant "SpeedDemon" (No. 18/SIRG/5508).

\section{APPENDIX: NUMERICAL CALCULATION OF TRANSMISSION AND REFLECTION COEFFICIENTS}

Here we will discuss how to numerically solve the stationary Schrödinger equation for the two-level system by the invariant imbedding method [53,54].

Let the potential $\mathcal{V}(x)$ be nonzero in the region $-d<x<$ $d$. We introduce the following dimensionless variables: $\bar{k}=$ $(2 m E)^{1 / 2} 2 d / \hbar, \quad \bar{x}=x /(2 d)+1 / 2, \quad \bar{\Omega}(\bar{x})=\left(4 m d^{2} / \hbar\right) \Omega(x)$ and $\bar{\Gamma}=\left(4 m d^{2} / \hbar\right)(\gamma-2 i \Delta)$. The non-Hermitian dimensionless Hamiltonian for the system takes the form

$$
\begin{aligned}
\overline{\mathcal{H}} & =\overline{\mathcal{H}}_{0}+\overline{\mathcal{V}}(\bar{x}), \\
\overline{\mathcal{H}}_{0} & =-\frac{\partial^{2}}{\partial \bar{x}^{2}}+\left(\begin{array}{cc}
0 & 0 \\
0 & -i \bar{\Gamma}
\end{array}\right), \\
\overline{\mathcal{V}}(\bar{x}) & =\left(\begin{array}{cc}
0 & \bar{\Omega}(\bar{x}) \\
\bar{\Omega}(\bar{x})^{*} & 0
\end{array}\right) .
\end{aligned}
$$

To set the matrices. we use as in the main text the convention for internal states $|1\rangle=\left(\begin{array}{l}1 \\ 0\end{array}\right)$ and $|2\rangle=\left(\begin{array}{l}0 \\ 1\end{array}\right)$. To simplify the notation, we will from now on drop the bars above variables and operators for the remaining part of this section. The corresponding stationary Schrödinger equation is now

$$
\begin{aligned}
& k^{2} \psi^{(1)}(x)=-\frac{\partial^{2}}{\partial x^{2}} \psi^{(1)}(x)+\Omega(x) \psi^{(2)}(x), \\
& k^{2} \psi^{(2)}(x)=-\frac{\partial^{2}}{\partial x^{2}} \psi^{(2)}(x)+\Omega(x)^{*} \psi_{1}(x)-i \Gamma \psi^{(2)}(x) .
\end{aligned}
$$

Let us denote as $\left|\Psi_{\alpha}(x)\right\rangle$ the wave vector for the atom impinging in internal level $\alpha, \alpha=1,2$. This vector has ground and excited state components, generically $\left\langle\beta \mid \psi_{\alpha}(x)\right\rangle, \beta=1,2$, which are still functions of $x$. We can define the matrices $F(x)$ and $\widetilde{F}(x)$ as

$$
F_{\beta, \alpha}(x)=\left\langle\beta \mid \psi_{\alpha}(x)\right\rangle, \quad \widetilde{F}_{\beta, \alpha}(x)=\left\langle\beta \mid \widetilde{\psi}_{\alpha}(x)\right\rangle,
$$

so the stationary Schrödinger equation can be rewritten as

$$
\begin{aligned}
& {\left[k^{2}-\mathcal{H}_{0}-\mathcal{V}(x)\right] F(x)=0,} \\
& {\left[k^{2}-\mathcal{H}_{0}-\mathcal{V}(x)\right] \widetilde{F}(x)=0 .}
\end{aligned}
$$

\section{Free motion, $\mathcal{V}=0$}

When $\mathcal{V}(x)=0$ we get

$$
\begin{aligned}
& {\left[k^{2}-\mathcal{H}_{0}\right]\left|\psi_{\alpha}(x)\right\rangle=0,} \\
& {\left[k^{2}-\mathcal{H}_{0}\right]\left|\widetilde{\psi}_{\alpha}(x)\right\rangle=0,}
\end{aligned}
$$

for $\alpha=1,2$. We can write down the solutions for particles "coming" from the left $\left|\psi_{\alpha}(x)\right\rangle$ in internal state $|\alpha\rangle$ as

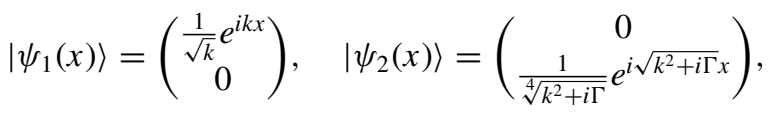

where we assume the branch $\operatorname{Im} \sqrt{k^{2}+i \Gamma} \geqslant 0 .\left|\psi_{2}(x)\right\rangle$ is a regular traveling wave only for real $\sqrt{k^{2}+i \Gamma}$ ). If the square root has an imaginary part, $\left|\psi_{2}(x)\right\rangle$ decays from left to right. The solutions for incidence from the right $\left|\widetilde{\psi}_{\alpha}(x)\right\rangle$ in internal state $|\alpha\rangle$ are similarly

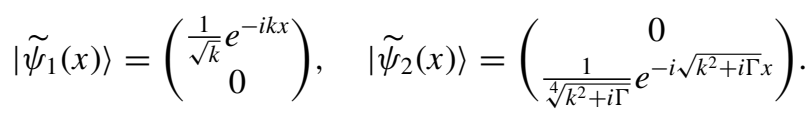

The normalization is chosen in such a way that the dimensionless probability current is constant (and equal) for all solutions with real $\sqrt{k^{2}+i \Gamma}$.

The solutions are given by $F(x)=h_{+}(x)$ and $\widetilde{F}(x)=$ $h_{-}(x)$, where

$$
h_{ \pm}(x)=\left(\begin{array}{cc}
\frac{1}{\sqrt{k}} e^{ \pm i k x} & 0 \\
0 & \frac{1}{\sqrt[4]{k^{2}+i \Gamma}} e^{ \pm i \sqrt{k^{2}+i \Gamma} x}
\end{array}\right) .
$$

The Wronskian is $W\left(h_{+}, h_{-}\right)(x)=2 i$ so that these are linearly independent solutions.

\section{General case}

To solve the general case, we construct the Green's function defined by

$$
\left(k^{2}-\mathcal{H}_{0}\right) G_{0}\left(x, x^{\prime}\right)=\delta\left(x-x^{\prime}\right) \mathbf{1} .
$$

It is given by

$$
\begin{aligned}
G_{0}\left(x, x^{\prime}\right) & =W^{-1} \begin{cases}h_{+}(x) h_{-}\left(x^{\prime}\right) & x>x^{\prime}, \\
h_{+}\left(x^{\prime}\right) h_{-}(x) & x^{\prime}>x,\end{cases} \\
& =-\frac{i}{2}\left(\begin{array}{cc}
\frac{1}{k} e^{i k\left|x-x^{\prime}\right|} & 0 \\
0 & \frac{e^{i \sqrt{k^{2}+i \Gamma}\left|x-x^{\prime}\right|}}{\sqrt{k^{2}+i \Gamma}}
\end{array}\right) .
\end{aligned}
$$


The Green's function allows us to solve for $F(x)$ and $\widetilde{F}(x)$ in integral form,

$$
\begin{aligned}
& F(x)=h_{+}(x)+\int_{-\infty}^{\infty} d x^{\prime} G_{0}\left(x, x^{\prime}\right) \mathcal{V}\left(x^{\prime}\right) F\left(x^{\prime}\right) \\
& \widetilde{F}(x)=h_{-}(x)+\int_{-\infty}^{\infty} d x^{\prime} G_{0}\left(x, x^{\prime}\right) \mathcal{V}\left(x^{\prime}\right) \widetilde{F}\left(x^{\prime}\right)
\end{aligned}
$$

\section{Asymptotic form of the solutions}

From Eq. (A10), we find the following asymptotic forms of $F(x)$ and $\widetilde{F}(x)$ :

$$
\begin{aligned}
& F_{\eta}(x)= \begin{cases}h_{+}(x)+h_{-}(x) R & x<0 \\
h_{+}(x) T & x>1\end{cases} \\
& \widetilde{F}_{\eta}(x)=\left\{\begin{array}{ll}
h_{-}(x) \widetilde{T} \\
h_{-}(x)+h_{+}(x) \widetilde{R} & x>0
\end{array},\right.
\end{aligned}
$$

where the $R$ and $T$ matrices for incidence from the left are given by

$$
\begin{aligned}
& R=W^{-1} \int_{0}^{1} d x^{\prime} h_{+}\left(x^{\prime}\right) \mathcal{V}\left(x^{\prime}\right) F\left(x^{\prime}\right), \\
& T=\mathbf{1}+W^{-1} \int_{0}^{1} d x^{\prime} h_{-}\left(x^{\prime}\right) \mathcal{V}\left(x^{\prime}\right) F\left(x^{\prime}\right),
\end{aligned}
$$

whereas, for right incidence,

$$
\begin{aligned}
& \widetilde{R}=W^{-1} \int_{0}^{\eta} d x^{\prime} h_{-}\left(x^{\prime}\right) \mathcal{V}\left(x^{\prime}\right) \widetilde{F}_{\eta}\left(x^{\prime}\right), \\
& \widetilde{T}=\mathbf{1}+W^{-1} \int_{0}^{\eta} d x^{\prime} h_{+}\left(x^{\prime}\right) \mathcal{V}\left(x^{\prime}\right) \widetilde{F}_{\eta}\left(x^{\prime}\right) .
\end{aligned}
$$

In particular, for left incidence in the ground-state, we get if $x<0$,

$$
\left|\psi_{1}(x)\right\rangle=\left(\begin{array}{c}
\frac{1}{\sqrt{k}} e^{i k x} \\
0
\end{array}\right)+\left(\begin{array}{c}
R_{1,1} \frac{1}{\sqrt{k}} e^{-i k x} \\
R_{2,1} \frac{1}{\sqrt[4]{k^{2}+i \Gamma}} e^{-i \sqrt{k^{2}+i \Gamma} x}
\end{array}\right),
$$

and, if $x>1$,

$$
\left|\psi_{1}(x)\right\rangle=\left(\begin{array}{c}
T_{1,1} \frac{1}{\sqrt{k}} e^{i k x} \\
T_{2,1} \frac{1}{\sqrt[4]{k^{2}+i \Gamma}} e^{i \sqrt{k^{2}+i \Gamma} x}
\end{array}\right) .
$$

When $\sqrt{k^{2}+i \Gamma}$ is real, the elements of $T$ and $R$ in Eqs. (A14) and (A15) are transmission and reflection amplitudes for waves traveling away from the interaction region. However when $\operatorname{Im} \sqrt{k^{2}+i \Gamma}>0$ the waves for the excited state 2 are evanescent. In scattering theory parlance the channel is "closed," so the $T_{2,1}$ and $R_{2,1}$ are just proportionality factors rather than proper transmission and reflection amplitudes for traveling waves. By continuity however, it is customary to keep the same notation and even terminology for closed or open channels.

In a similar way, for right incidence in the ground state and $x>1$,

$$
\left|\widetilde{\psi}_{1}(x)\right\rangle=\left(\begin{array}{c}
\frac{1}{\sqrt{k}} e^{-i k x} \\
0
\end{array}\right)+\left(\begin{array}{c}
\widetilde{R}_{1,1} \frac{1}{\sqrt{k}} e^{i k x} \\
\widetilde{R}_{2,1} \frac{1}{\sqrt[4]{k^{2}+i \Gamma}} e^{i \sqrt{k^{2}+i \Gamma x}}
\end{array}\right),
$$

whereas, for $x<0$,

$$
\left|\widetilde{\psi}_{1}(x)\right\rangle=\left(\begin{array}{c}
\widetilde{T}_{1,1} \frac{1}{\sqrt{k}} e^{-i k x} \\
\widetilde{T}_{2,1} \frac{1}{\sqrt[4]{k^{2}+i \Gamma}} e^{-i \sqrt{k^{2}+i \Gamma} x}
\end{array}\right)
$$

Note that alternative definitions of the amplitudes may be found in many works, without momentum prefactors.

The amplitudes relevant for the main text are $T^{l}=T_{1,1}$, $T^{r}=\widetilde{T}_{1,1}, R^{l}=R_{1,1}$, and $R^{r}=\widetilde{R}_{1,1}$. The following section explains how to compute them.

\section{Differential equations for $R$ and $\boldsymbol{T}$ matrices}

To solve for $R$ and $T$ we will use cutoff versions of the potential,

$$
\mathcal{V}_{\eta}(x)= \begin{cases}\mathcal{V}(x) & 0 \leqslant x \leqslant \eta \\ 0 & \text { otherwise }\end{cases}
$$

where $0 \leqslant \eta \leqslant 1$, and corresponding matrices

$$
\begin{aligned}
& R_{\eta}=W^{-1} \int_{0}^{\eta} d x^{\prime} h_{+}\left(x^{\prime}\right) \mathcal{V}\left(x^{\prime}\right) F_{\eta}\left(x^{\prime}\right), \\
& T_{\eta}=\mathbf{1}+W^{-1} \int_{0}^{\eta} d x^{\prime} h_{-}\left(x^{\prime}\right) \mathcal{V}\left(x^{\prime}\right) F_{\eta}\left(x^{\prime}\right), \\
& \widetilde{R}_{\eta}=W^{-1} \int_{0}^{\eta} d x^{\prime} h_{-}\left(x^{\prime}\right) \mathcal{V}\left(x^{\prime}\right) \widetilde{F}_{\eta}\left(x^{\prime}\right), \\
& \widetilde{T}_{\eta}=\mathbf{1}+W^{-1} \int_{0}^{\eta} d x^{\prime} h_{+}\left(x^{\prime}\right) \mathcal{V}\left(x^{\prime}\right) \widetilde{F}_{\eta}\left(x^{\prime}\right) .
\end{aligned}
$$

Taking the derivative of these matrices with respect to $\eta$, we find a set of four coupled differential equations,

$$
\begin{aligned}
& \frac{d R_{\eta}}{d \eta}=W^{-1} \widetilde{T}_{\eta} h_{+}(\eta) \mathcal{V}(\eta) h_{+}(\eta) T_{\eta} \\
& \frac{d T_{\eta}}{d \eta}=W^{-1}\left[h_{-}(\eta)+\widetilde{R}_{\eta} h_{+}(\eta)\right] \mathcal{V}(\eta) h_{+}(\eta) T_{\eta} \\
& \frac{d \widetilde{R}_{\eta}}{d \eta}=W^{-1}\left[h_{-}(\eta)+\widetilde{R}_{\eta} h_{+}(\eta)\right] \mathcal{V}(\eta)\left[h_{-}(\eta)+h_{+}(\eta) \widetilde{R}_{\eta}\right] \\
& \frac{d \widetilde{T}_{\eta}}{d \eta}=W^{-1} \widetilde{T}_{\eta} h_{+}(\eta) \mathcal{V}(\eta)\left[h_{-}(\eta)+h_{+}(\eta) \widetilde{R}_{\eta}\right]
\end{aligned}
$$

The initial conditions are $R_{0}=\widetilde{R}_{0}=0$ and $T_{0}=\widetilde{T}_{0}=\mathbf{1}$.

\section{Improving numerical efficiency}

The Eqs. (A22) and (A23) involve only matrices for incidence from the right, they do not couple to any left-incidence matrix, whereas the equations for left incidence amplitudes involve couplings with amplitudes for right incidence. This asymmetry is due to the way we do the potential slicing. The asymmetry is not "fundamental" but we can use it for our advantage to simplify calculations. We can solve Eqs. (A22) and (A23) to get amplitudes for right incidence. To get amplitudes for left incidence we use a mirror image of the potential and solve also these two equations. Thus it is enough to find an efficient numerical method to solve Eqs. (A22) and (A23). In 
principle, one can now solve these differential equations from $\eta=0$ to 1 to get all reflection and transmission amplitudes using the boundary conditions $\widetilde{R}_{0}=0$ and $\widetilde{T}_{0}=\mathbf{1}$. However, due to the exponential nature of the free-space solutions $h_{ \pm}(x)$ especially if $\operatorname{Im} \sqrt{k^{2}+i \Gamma}>0$, this is not very efficient numerically.

To avoid this problem, we make new definitions:

$$
\begin{aligned}
\hat{S}_{\eta} & =\mathbf{1}+h_{+}(\eta) \widetilde{R}_{\eta} h_{-}^{-1}(\eta), \\
\hat{T}_{\eta} & =h_{+}(0) \widetilde{T}_{\eta} h_{-}^{-1}(\eta), \\
\hat{\mathcal{V}}(\eta) & =W^{-1} h_{+}^{2}(0) \mathcal{V}(\eta), \\
\hat{Q} & =i h_{+}^{-2}(0) .
\end{aligned}
$$

Rewriting Eqs. (A22) and (A23) in terms of these new variables, we get

$$
\begin{aligned}
& \frac{d \hat{S}_{\eta}}{d \eta}=-2 \hat{Q}+\hat{Q} \hat{S}_{\eta}+\hat{S}_{\eta}\left[\hat{Q}+\hat{\mathcal{V}}(\eta) \hat{S}_{\eta}\right], \\
& \frac{d \hat{T}_{\eta}}{d \eta}=\hat{T}_{\eta}\left[\hat{Q}+\hat{\mathcal{V}}(\eta) \hat{S}_{\eta}\right],
\end{aligned}
$$

with initial conditions $\hat{T}_{0}=\hat{S}_{0}=\mathbf{1}$.

Let us consider solely incidence in the ground state. For right incidence in the ground state, the reflection coefficients and transmission coefficient are

$$
\begin{aligned}
& \widetilde{R}_{1,1}=e^{-2 i k}\left[\left(\hat{S}_{\eta=1}\right)_{1,1}-1\right], \\
& \widetilde{R}_{2,1}=\frac{\sqrt[4]{k^{2}+i \Gamma}}{\sqrt{k}} e^{-i k-i \sqrt{k^{2}+i \Gamma}}\left(\hat{S}_{\eta=1}\right)_{2,1},
\end{aligned}
$$

$$
\begin{aligned}
& \widetilde{T}_{1,1}=e^{-i k}\left(\hat{T}_{\eta=1}\right)_{1,1} \\
& \widetilde{T}_{2,1}=\frac{\sqrt[4]{k^{2}+i \Gamma}}{\sqrt{k}} e^{-i k}\left(\hat{T}_{\eta=1}\right)_{2,1} .
\end{aligned}
$$

\section{Bounds from unitarity}

The $S$ matrix

$$
S=\left(\begin{array}{cccc}
T_{11} & T_{12} & \widetilde{R}_{11} & \widetilde{R}_{12} \\
T_{21} & T_{22} & \widetilde{R}_{21} & \widetilde{R}_{22} \\
R_{11} & R_{12} & \widetilde{T}_{11} & \widetilde{T}_{12} \\
R_{21} & R_{22} & \widetilde{T}_{21} & \widetilde{T}_{22}
\end{array}\right)
$$

is unitary for Hermitian Hamiltonians, in particular, when $\gamma=$ 0 . Unitarity implies relations among the matrix elements and in particular

$$
\begin{aligned}
& 1 \geqslant\left|R_{11}\right|^{2}+\left|T_{11}\right|^{2}, \\
& 1 \geqslant\left|\widetilde{R}_{11}\right|^{2}+\left|\widetilde{T}_{11}\right|^{2}, \\
& 1 \geqslant\left|\widetilde{R}_{11}\right|^{2}+\left|T_{11}\right|^{2}, \\
& 1 \geqslant\left|R_{11}\right|^{2}+\left|\widetilde{T}_{11}\right|^{2} .
\end{aligned}
$$

While the first two Eqs. (A28) and (A29) are rather obvious because of probability conservation, the last two Eqs. (A30) and (A31) are less so, and set physical limits to the possible asymmetric devices that can be constructed in the groundstate subspace.
[1] J. C. Maxwell, Theory of Heat, 4th ed. (Longmans, Green and Co., London, 1875), pp. 328-329.

[2] Maxwell's Demon: Entropy, Information, Computing, edited by H. S. Leff and A. Rex (Princeton University Press, Princeton, 1990).

[3] A. Ruschhaupt and J. G. Muga, Phys. Rev. A 70, 061604(R) (2004).

[4] A. Ruschhaupt and J. G. Muga, Phys. Rev. A 73, 013608 (2006).

[5] A. Ruschhaupt, J. G. Muga, and M. G. Raizen, J. Phys. B 39, L133 (2006).

[6] A. Ruschhaupt and J. G. Muga, Phys. Rev. A 76, 013619 (2007).

[7] M. G. Raizen, A. M. Dudarev, Qian Niu, and N. J. Fisch, Phys. Rev. Lett. 94, 053003 (2005).

[8] A. M. Dudarev, M. Marder, Qian Niu, N. J. Fisch, and M. G. Raizen, Europhys. Lett. 70, 761 (2005).

[9] A. Ruschhaupt, J. G. Muga, and M. G. Raizen, J. Phys. B 39, 3833 (2006).

[10] M. Raizen, Science 324, 1403 (2009).

[11] M. Jerkins, I. Chavez, U. Even, and M. G. Raizen, Phys. Rev. A 82, 033414 (2010).

[12] J. G. Muga, J. P. Palao, B. Navarro, and I. L. Egusquiza, Phys. Rep. 395, 357 (2004), Eq. (113) should read $\left\langle x|V| x^{\prime}\right\rangle=$ $\left\langle-x|V|-x^{\prime}\right\rangle^{*}$.

[13] A. Mostafazadeh, Scattering theory and PT-symmetry, in Parity-time Symmetry and Its Applications, edited by D. Christodoulides, J. Yang, Springer Tracts in Modern Physics Vol 280 (Springer, Singapore, 2018).
[14] A. Ruschhaupt, F. Delgado, and J. G. Muga, J. Phys. A 38, L171 (2005).

[15] S. Longhi, Europhys. Lett. 120, 64001 (2017).

[16] V. V. Konotop, J. Yang, and D. A. Zezyulin, Rev. Mod. Phys. 88, 035002 (2016).

[17] N. Moiseyev, Non-Hermitian Quantum Mechanics (Cambridge U. Press, Cambridge 2011).

[18] H. Feshbach, Ann. Phys. (NY) 5, 357 (1958).

[19] A. Ruschhaupt, J. A. Damborenea, B. Navarro, J. G. Muga, and G. C. Hegerfeldt, Europhys. Lett. 67, 1 (2004).

[20] C. M. Bender and S. Boettcher, Phys. Rev. Lett. 80, 5243 (1998).

[21] M. Znojil, in Non-Selfadjoint Operators in Quantum Physics, edited by F. Bagarello, J.-P. Gazeau, F. H. Szafraniec, and M. Znojil (Wiley, Hoboken, New Jersey, 2015), Ch. 1, p. 7.

[22] S. Longhi, J. Phys. A: Math. Theor. 47, 485302 (2014).

[23] S. Nixon and J. Yang, Phys. Rev. A 93, 031802(R) (2016).

[24] S. Nixon and J. Yang, Opt. Lett. 41, 2747 (2016).

[25] P. Chen and Y. D. Chong, Phys. Rev. A 95, 062113 (2017).

[26] A. Ruschhaupt, T. Dowdall, M. A. Simón, and J. G. Muga, Europhys. Lett. 120, 20001 (2017).

[27] M. A. Simón, A. Buendía, and J. G. Muga, Mathematics 6, 111 (2018).

[28] M. A. Simón, A. Buendía, A. Kiely, A. Mostafazadeh, and J. G. Muga, Phys. Rev. A 99, 052110 (2019).

[29] A. Alaña, S. Martínez-Garaot, M. A. Simón, and J. G. Muga, J. Phys. A 53, 135304 (2020). 
[30] D. Bernard and A. LeClair, in Statistical Field Theories, edited by A. Cappelli and G. Mussardo, NATO Science Series Vol 73, (Springer, Dordrecht, 2002), pp. 207-214.

[31] K. Kawabata, K. Shiozaki, M. Ueda, and M. Sato, Phys. Rev. X 9, 041015 (2019).

[32] A. Mostafazadeh, Int. J. Geom. Methods Mod. Phys. 07, 1191 (2010).

[33] Z. Lin, H. Ramezani, T. Eichelkraut, T. Kottos, H. Cao, and D. N. Christodoulides, Phys. Rev. Lett. 106, 213901 (2011).

[34] L. Feng et al., Science 333, 729 (2011).

[35] S. Fan et al., Science 335, 38 (2012).

[36] B. Peng et al., Nat. Phys. 10, 394 (2014).

[37] R. J. Potton, Rep. Prog. Phys. 67, 717 (2004).

[38] L. Deák and T. Fülöp, Ann. Phys. 327, 1050 (2012).

[39] Y.-L. Xu, L. Feng, M.-H. Lu, and Y.-F. Chen, IEEE Photonics J. 6, 0600507 (2014).

[40] A. Ruschhaupt, J. G. Muga, and G. C. Hegerfeldt, Lect. Notes Phys. 789, 65 (2009).

[41] G. C. Hegerfeldt and D. G. Sondermann, Quantum Semiclass. Opt. 8, 121 (1996).

[42] J. A. Damborenea, I. L. Egusquiza, G. C. Hegerfeldt, and J. G. Muga, Phys. Rev. A 66, 052104 (2002).
[43] B. Navarro, I. L. Egusquiza, J. G. Muga, and G. C. Hegerfeldt, Phys. Rev. A 67, 063819 (2003).

[44] M. K. Oberthaler, R. Abfalterer, S. Bernet, J. Schmiedmayer, and A. Zeilinger, Phys. Rev. Lett. 77, 4980 (1996).

[45] E. W. Streed, J. Mun, M. Boyd, G. K. Campbell, P. Medley, W. Ketterle, and D. E. Pritchard, Phys. Rev. Lett. 97, 260402 (2006).

[46] J. Zhang et al., Phys. Rev. Lett. 110, 240501 (2013).

[47] H. Feshbach, Ann. Phys. (NY) 19, 287 (1962).

[48] R. D. Levine, Quantum Mechanics of Molecular Rate Processes (Oxford University Press, London, 1969).

[49] D. O. Chudesnikov and V. P. Yakovlev, Laser Phys. 1, 110 (1991).

[50] N. Khaneja, T. Reiss, C. Kehlet, T. Schulte-Herbrüggen, and S. J. Glaser, J. Magn. Reson. 172, 296 (2005).

[51] N. Wu, A. Nanduri, and H. Rabitz, Phys. Rev. B 91, 041115(R) (2015).

[52] M. Zeyen, Focused raman beam addressing for a trapped-ion quantum processor, Master Thesis, ETH, Zurich, 2016.

[53] S. Singer, K. F. Freed, and Y. B. Band, J. Chem. Phys. 77, 1942 (1982).

[54] Y. B. Band and I. Tuvi, J. Chem. Phys. 100, 8869 (1994). 